\title{
CALCULATION OF RESONANT FREQUENCY OF HEXAGONAL SPLIT RING RESONATOR USING ANN
}

\author{
Anuradha Singh ${ }^{1}$, Sanjay Kumar Sharma ${ }^{2}$ \\ ${ }^{1}$ Electronics and Communication Department, Babu Banarasi Das University, Lucknow \\ ${ }^{2}$ Electronics and Communication Department, Babu Banarasi Das University, Lucknow
}

\begin{abstract}
In this paper, an artificial neural network model is proposed to find out the resonant frequency of a metamaterial based Hexagonal Split Ring Resonator (SRR). The main advantage of Hexagonal SRR is small electrical size at resonance (electrically small) in the microwave regime. The method can be used for a wide range of different depending parameters (effective radius of the Hexagonal ring, split gap in the rings, width of the rings, spacing between the rings, depth of the substrate). The calculated resonant frequency is in very good agreement with the experimental results.
\end{abstract}

Keywords- Metamaterial, Hexagonal Split Ring Resonator, Artificial Neural Network, Resonant Frequency ****

\section{INTRODUCTION AND BACKGROUND}

The electric and magnetic properties of materials are determined by two important material parameters, dielectric permittivity $(\varepsilon)$ and magnetic permeability $(\mu)$. It is shown in fig.1. In 1968, Victor Veselago [1] predicted that $\varepsilon$ and $\mu$ can also be negative.

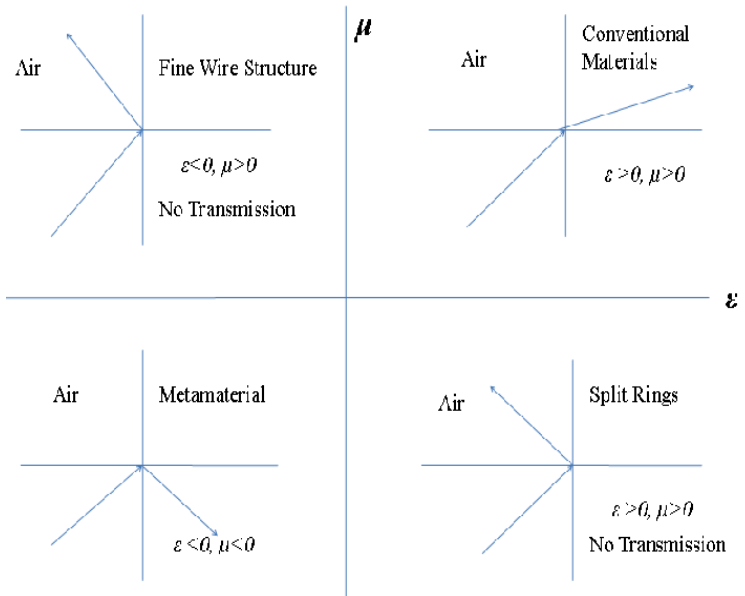

Fig.1. Classification of Materials

When both permittivity \& permeability of any material are greater than zero $(\varepsilon>0, \mu>0)$ then such material are called as Conventional materials When permittivity is less than zero $\&$ permeability is greater than zero $(\varepsilon<0, \mu>0)$, then propagation of wave is not possible because $n(n= \pm \sqrt{\varepsilon \mu})$ is imaginary in this condition. Such type of condition can be implemented using fine wire structures.

When permittivity is greater than zero \& permeability is less than zero $(\varepsilon>0, \mu<0)$, then again propagation of wave is not possible because $n$ is imaginary. Such type of condition can be implemented using Split Ring Resonators.
When permittivity \& permeability are less than zero $(\varepsilon<0$, $\mu<0$ ), then propagation of wave is possible because $n$ is real. Such type of condition can be implemented by fine wire and split ring resonator. This combined structure is called Metamaterial.

\section{METAMATERIAL AND HEXAGONAL SPLIT RING RESONATOR}

Metamaterials are artificial materials engineered to have properties that may not be found in nature. The Metamaterials are usually arranged in periodic patterns.

Metamaterial structure composed of SRR and strip wires. Negative permeability can be achieved by using split ring resonator and negative permittivity can be achieved by using strip wire (Fig.2).

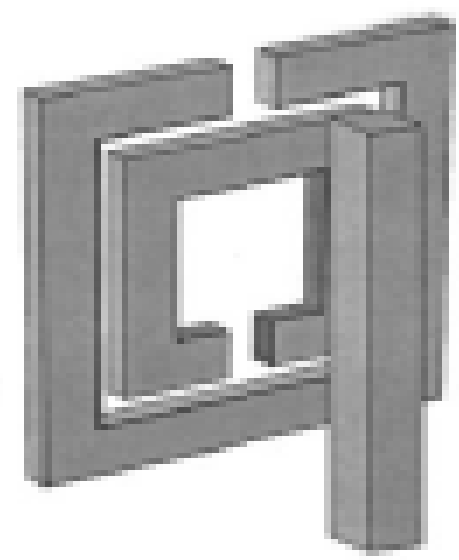

Fig.2. SRR and Strip Wire

SRR are common in the metamaterial design as they exhibit negative permittivity and permeability for frequencies close to their resonant frequency [2]. This structure consists of two concentric rings separated by a gap, both having splits at 
opposite sides. Magnetic resonance is induced by the splits at the rings and by the gap between inner and outer rings. SRR vary in their shape and structure such as square SRR [3] and circular SRR [4]. Rarer structures include multiple SRR [5], spiral SRR [6], triangular SRR [7] and elliptical SRR [8]. In the proposed work, neural network model is design to find out the resonant frequency for a new structure Hexagonal SRR.

Fig.3 (a, b) shows the schematic geometry of Hexagonal SRR and its equivalent circuit. In fig. 3(a), ' $a$ ' is radius of hexagon, ' $c$ ' is the width of the strip, the separation between the ring is ' $\mathrm{d}$ ', the split gap of both the rings are ' $\mathrm{g}_{1}$ ' and ' $\mathrm{g}_{2}$ ' , ' $h$ ' is the depth of the substrate. In equivalent circuit model of the Hexagonal SRR, inductance (L) is due to the rings. The capacitance $\left(\mathrm{C}_{\mathrm{g} 1}\right.$ and $\left.\mathrm{C}_{\mathrm{g} 2}\right)$ is due to the split gaps and $\mathrm{C}_{1}$ is the capacitance of upper half ring and $C_{2}$ is the capacitance of lower half ring [9].

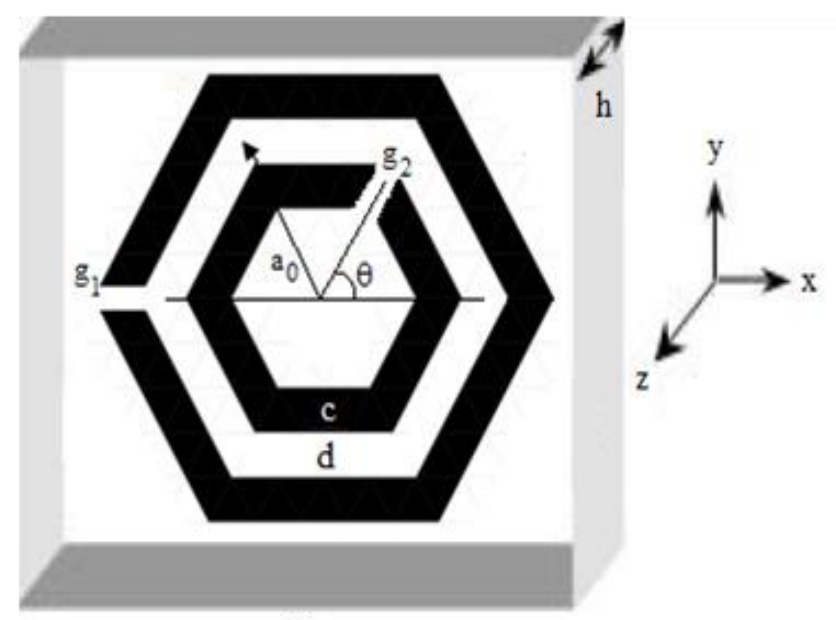

(a)

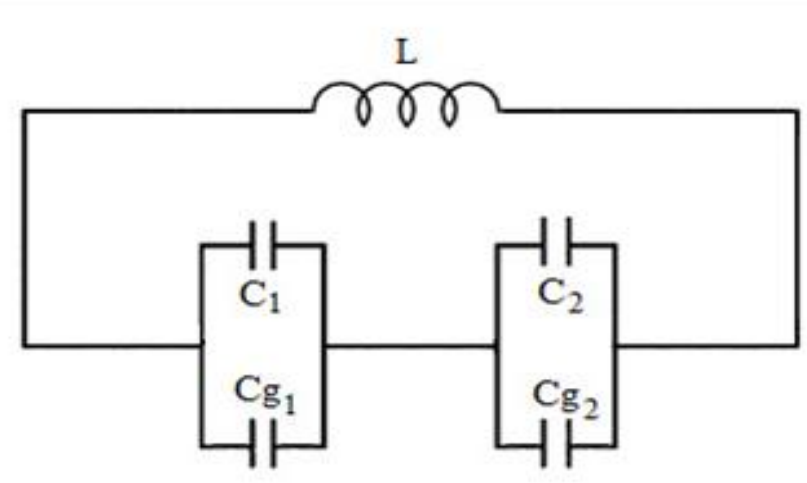

(b)

Fig.3. (a) Structure of Hexagonal SRR (b) Equivalent Circuit model of the Hexagonal SRR

The Resonant Frequency $\left(\mathrm{f}_{0}\right)$ [9] of the hexagonal SRR can be computed using the equation (1)-

$$
f_{0}=\frac{1}{\left.\left(2 \pi \sqrt{\left(2 a_{e q}\right.} \cdot L_{N e t} \cdot C_{N e t}\right)\right)}(1)
$$

Where $a_{\text {eq }}$ is the effective radius of Hexagonal SRR and its expression is [9]

$$
a_{e q}=2 a \cdot \sin \left\{\frac{\pi}{N}\right\}-\frac{g}{N}
$$

Here $\mathrm{N}=6$.

$\mathrm{L}_{\text {Net }}$ is the equivalent inductance and its expression is [9]:

$$
L_{N e t}=0.00508 *\left(2.303 \log _{10} \frac{4 l}{c}-2.636\right)
$$

Where $\mathrm{c}$ is the width of the strip, $l$ is the perimeter of the hexagonal SRR and the expression of $l$ is shown in the following equation [9]:

$$
l=2 \cdot a \cdot N \cdot \sin \frac{\pi}{N}
$$

$\mathrm{C}_{\mathrm{Net}}$ is the equivalent capacitance of the structure and the expression is [9]:

$$
C_{N e t}=\left\{\frac{\left(N \cdot \sin \left\{\frac{\pi}{N}\right\}+\beta\right)^{2}-\left(\frac{\Delta}{a}\right)^{2}}{2\left(N \cdot \sin \left\{\frac{\pi}{N}\right\}+\beta\right)}\right\}
$$

Where $\beta=C_{g} / a . C_{p u l}$ and $C_{p u l}$ is the capacitance per unit length of the hexagonal SRR (Appendix A) and

$$
\Delta=a \sin \left\{\frac{\pi}{N}\right\} \cdot(2 m+1)-a \cos \left\{\frac{\pi}{N}\right\} \cdot \tan \left\{\frac{\pi}{N}-\varphi\right\}
$$

Now the capacitance of the upper half-ring $\left(\mathrm{C}_{\mathrm{u}}\right.$ and lower half-ring $\left(\mathrm{C}_{\mathrm{l})}\right.$ can be easily computed from $\Delta$ and $\mathrm{C}_{\mathrm{pul}}$ as [9]:

And

$$
C_{u}=\left[N \cdot a \cdot \sin \left\{\frac{\pi}{N}\right\}-\Delta\right] \cdot C_{p u l}
$$

$$
C_{l}=\left[N \cdot a \cdot \sin \left\{\frac{\pi}{N}\right\}+\Delta\right] \cdot C_{p u l}
$$

$\mathrm{C}_{\mathrm{g}}$ is the capacitance due to the split gaps in the rings and can be estimated using the equation [9]:

$$
C_{g}=\frac{\varepsilon_{0} \cdot \varepsilon_{r} \cdot c \cdot h}{g}
$$

Where $\varepsilon_{0}$ is the permittivity of free space $\left(\varepsilon_{0}=8.8541 \times 10^{-12}\right.$ $\mathrm{F} / \mathrm{mt}$ ) and $\varepsilon_{\mathrm{r}}$ is the relative permittivity which is different for materials.

\section{IMPLEMENTATION OF ARTIFICIAL NEURAL NETWORK MODEL}

An artificial neural network is composed of many artificial neurons that are linked together according to specific network architecture. The main body of an artificial neuron is called a node or unit. Neural Network model can be trained with known data before tested for their capability on unknown data. 


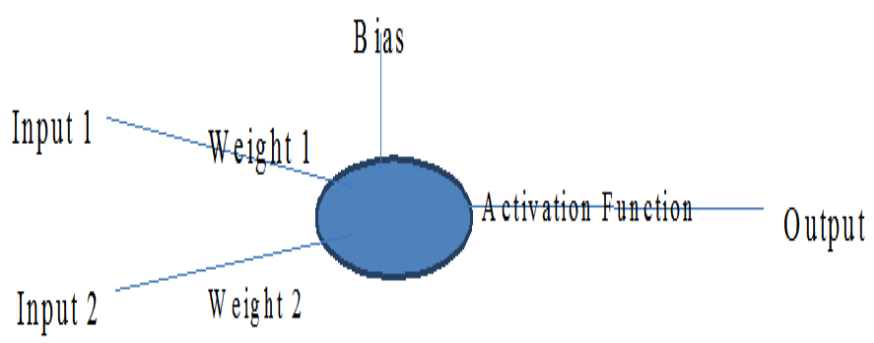

Fig.4. Artificial Neural Network

ANN is specified by an architecture which is a set of neurons and links connecting neurons. Each link has a weight which is the strength of the signal and bias is an external input for NN. An activation function defines the output of the node. In this paper, multi layer feed forward ANN architecture is used. A set of random value distributed uniformly between -1 and +1 are used to initialize the weight of the neural model.

Fig. 5 shows the multi layer feed forward ANN architecture to calculate the resonant frequency. There are five input nodes in the proposed ANN model: a is effective radius, $g$ is split gap in the conductor rings, $\mathrm{c}$ is width of the rings, $\mathrm{d}$ is separation between the rings of the Hexagonal Split Ring Resonator and $\mathrm{h}$ is depth of the substrate.

The best network's configuration in this work is 5-10-10-101. This means that the ANN model has three hidden layers and each hidden layer have ten neurons. In the output layer, there is only one neuron and output of this model is resonant frequency $\left(\mathrm{f}_{0}\right)$.

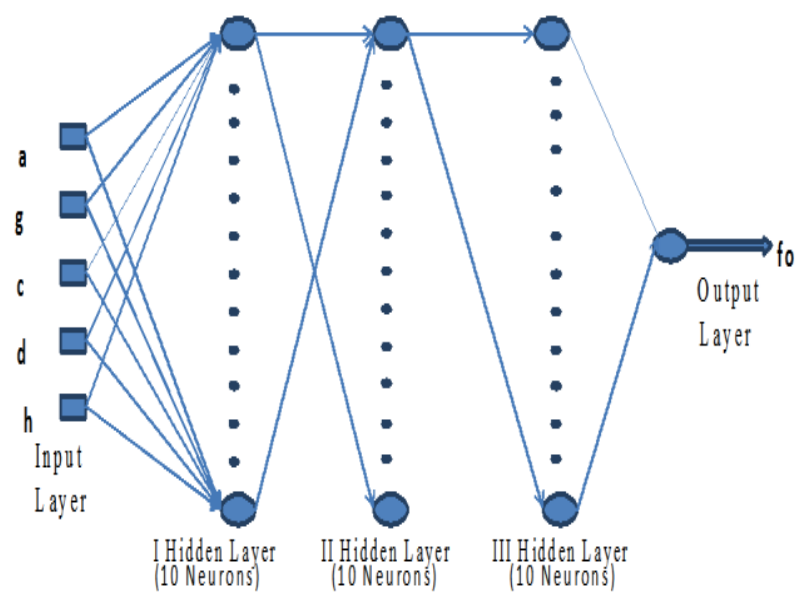

Fig.5. ANN model for Resonant Frequency

The Levenberg-Marquardt (LM) Back propagation is used for training purpose. Activation function used in first, second and third hidden layers are tangent sigmoidal and log sigmoidal respectively. In output layer, purelinear activation function is used. Total datasets are 124. These data sets are generated using the above equations. $75 \%$ of total dataset are used in training and rest $25 \%$ datasets are used in testing. Mean Square Error (MSE) of 9.65E-7 is used as the target to meet for 97 data sets
The status of trained model is shown in fig.6. This graph shows the convergence of ANN model. Total no. of epochs required to train the model is 1758 . The total time required to train the model is 1 minute 3 seconds. After each iteration, the error will be decrease and output of neural model is approaches to target.

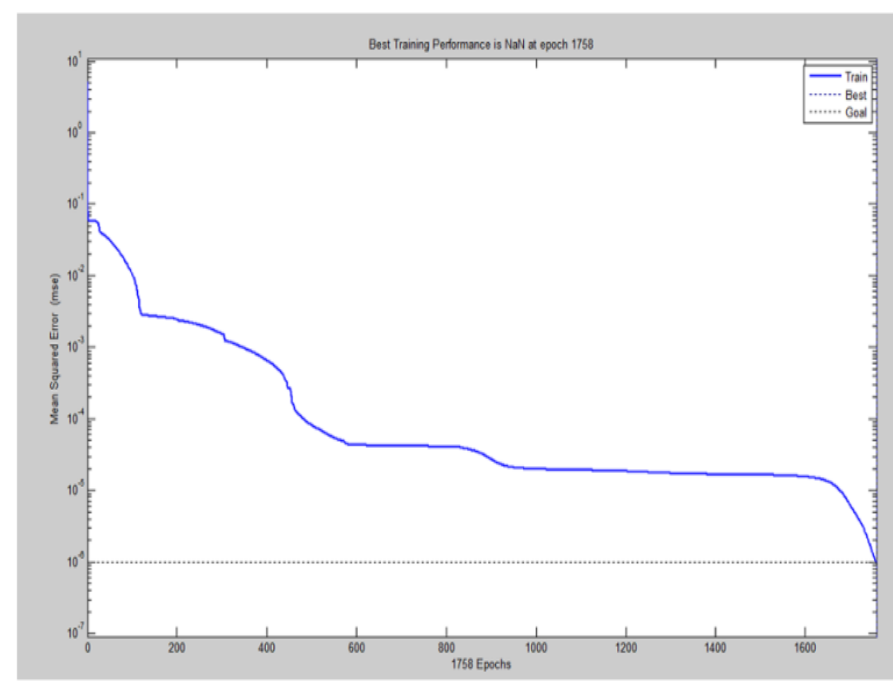

Fig.6. Convergence of the model during training of Resonant Frequency calculation

\section{RESULTS AND CONCLUSIONS}

The resonant frequency of Hexagonal Split Ring Resonator is shown in table 1 (Appendix B). The testing results are closer to the literature results. The absolute error in 27 testing data set is 1.10700 . This absolute error is very less. In this paper, ANN model is developed to calculate the resonant frequency of Hexagonal Split Ring Resonator. The training time for the model is about 1 minute 3 seconds and after training, the calculation time is less than 100 Pico seconds in real time calculations. Thus, the computation by neural model is very fast after training.

\section{REFERENCES}

[1]. V. G. Veselago, "The Electrodynamics of Substances with Simultaneously Negative Values of $\varepsilon$ and $\mu$ ", Soviet Physics Uspekhi, Vol. 10, No.4, 1968.

[2]. J.B. Pendry, A.J. Holden, W.J.Stewart, "Extremely low frequency plasmons in metallic Mesostructures", Phys. Rev. Lett., 1996.

[3]. C. Saha, J.Y. Siddiqui, Y. M. M. Antar, "Theoretical investigation of the square split ring resonator", Proceedings of URSI NA Radio Science Meet, 2007.

[4]. C. Saha, J. Y. Siddiqui, "Estimation of the resonance frequency of conventional \& rotational circular split ring resonators", IEEE Applied Electromagnetics Conference (AEMC), Kolkata, 2009.

[5]. Filiberto Bilotti, Alessandro Toscano, Lucio Vegni, Koray Aydin Kamil Boratay Alici, and Ekmel Ozbay, "Equivalent-Circuit Models for the Design of Metamaterials Based on Artificial Magnetic Inclusions", IEEE transactions on microwave theory and techniques, vol. 55, 2007. 
[6]. Filiberto Bilotti, Alessandro Toscano, and Lucio Vegni, "Design of Spiral and Multiple Split-Ring Resonators for the Realization of Miniaturized Metamaterial Samples", IEEE transactions on antennas and propagation, vol. 55, 2007.

[7]. Vidyalakshmi. M. R., Dr. S. Raghavan, “A CAD Model of Triangular Split Ring Resonator Based on Equivalent Circuit Approach", IEEE Applied Electromagnetics Conference (AEMC), Kolkata, 2009.

[8]. Vipul Sharma, S. S. Pattnaik, Tanuj Garg, Swapna Devi, "A Microstrip Metamaterial Split Ring Resonator, International Journal of Physical Sciences, 2011.

[9]. Sumanta Bose, M. Ramaraja, Dr. S. Raghavana, Swadhyaya Kumara, "Mathematical Modeling, Equivalent Circuit Analysis and Genetic Algorithm Optimization of an N-sided Regular Polygon Split ring Resonator (NRPSRR)", $2^{\text {nd }}$ International Conference on Communication, computing \& Security, Procedia Technology (6), 2012.

\section{APPENDIX}

\section{Appendix A}

The Capacitance per unit length of the Hexagonal SRR, $C_{\text {pul }}$ is estimated as:

$$
C_{p u l}=\varepsilon_{0} \cdot\left(\frac{\varepsilon_{r}+1}{2}\right) \cdot \frac{\varepsilon\left(\sqrt{1}-\sigma^{2}\right)}{\varepsilon(\sigma)}
$$

Where $\sigma=\frac{d}{d+2 c}$ and $\varepsilon($.$) is the complete elliptical integral of$ the second kind, defined as:

$$
\left.\varepsilon(k)=\int_{0}^{\frac{\pi}{2}} \sqrt{(1}-(k \sin \theta)^{2} d \theta\right)
$$

\section{Appendix B}

Table 1- Comparison of resonant frequency calculate by conventional method and neural network method

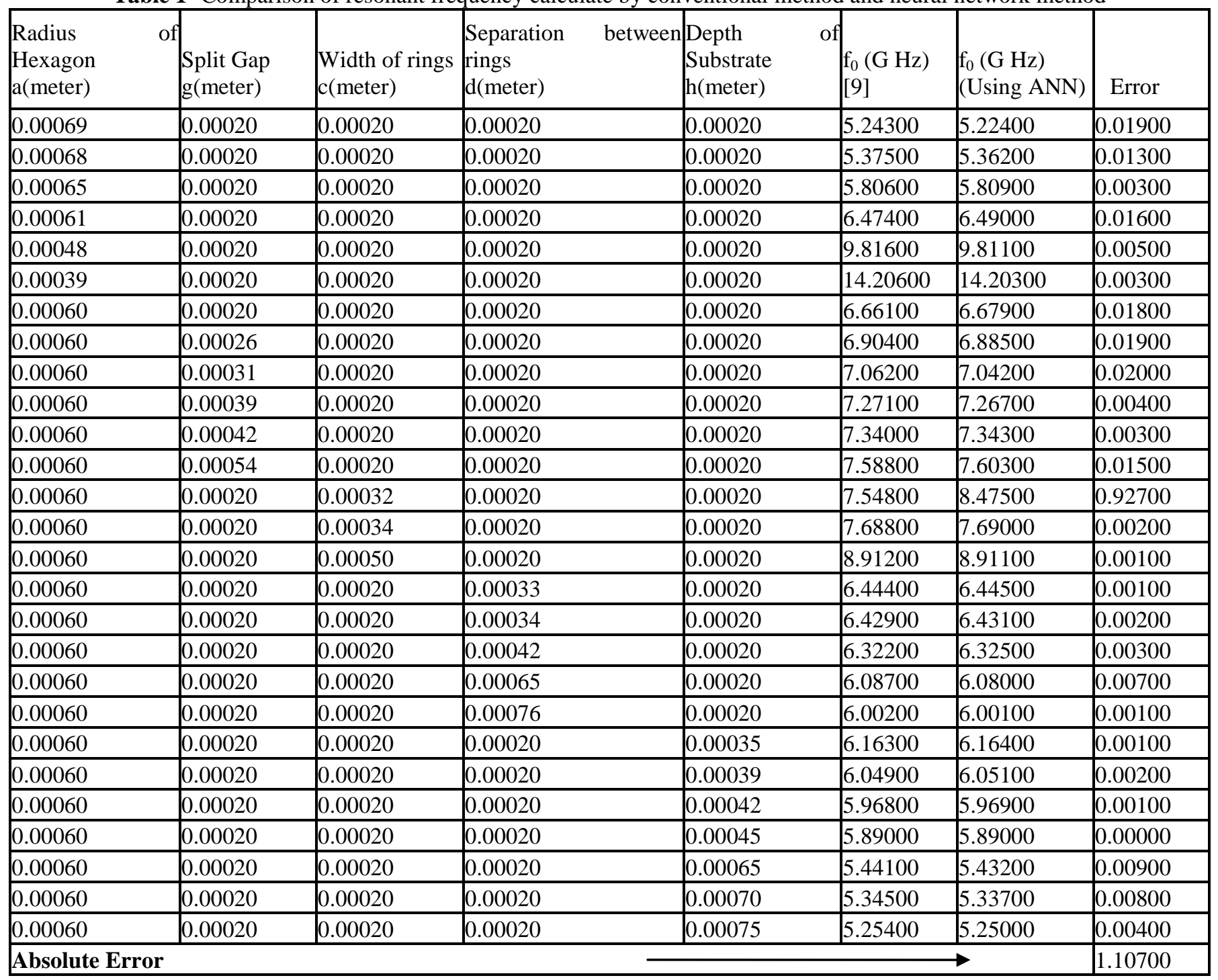

